\title{
A Novel and Cost EfFective Approach to Public Vehicle Tracking System
}

\author{
Deepak Mishra $^{1}$, Apurv Vasal $^{2}$, Puneet Tandon ${ }^{1}$ \\ ${ }^{1}$ PDPM Indian Institute of Information Technology, Design and Manufacturing, \\ Jabalpur, Madhya Pradesh, India \\ ${ }^{2}$ Space Applications Centre (ISRO), Ahmedabad, Gujarat, India \\ deemish@iittdmj.ac.in, apurv@sac.isro.gov.in, ptandon@iittdmj.ac.in
}

\begin{abstract}
There are lots of efforts being made by public transport corporations to improve public vehicle occupancy by requesting the public to use public transport over other modes of transportation. It can be noted that if the passenger knows with high confidence that the bus is going to come, he/she will definitely wait rather than opting for other modes of transport. Efficient information can therefore help the users to choose faster and more easier connections which saves their time. Trends in wireless technology like Global System for Mobile communication (GSM) and Radio Frequency Identification (RFID) have resulted in easier and faster communication. This paper presents a vehicle tracking system by integrating both of the above mentioned technologies. The central server uses geographic information system (GIS) to track vehicles to display the position information on the electronic map. This paper suggests a new cost-effective way for tracking a vehicle in public transport domain and compares proposed technique to some of the methods advised previously for tracking public vehicles.
\end{abstract}

\section{KEYWORDS}

Public Vehicle Tracking, GSM, RFID

\section{INTRODUCTION}

One of the major problems faced by public transport systems is low occupancy rate. One reason for this is that a passenger normally does not know the public vehicle scheduling information and its arrival timing information at predefined stops. Thus, it is possible to motivate a passenger to opt for public vehicles having predefined routes by providing the vehicle location and time of arrival at respective stoppage. Large cities with fleet of vehicles require a system to determine location of movement of passenger vehicles at a given time. The future intelligent transportation system (ITS) will rely predominantly on several vehicle communication systems [1] including peer-to-peer and peer-to-base station communications. Thus, this system provides the facility to know about the time for arrival at different stops and the location of public vehicles having predefined routes. This paper introduces a system that:

(i) provides a very cost-effective solution as compared to existing technologies for tracking public vehicles, and 
(ii) offers an open architecture that can be easily expanded to other applications.

In this paper, we have dealt with vehicles travelling in 2D object space. In a two-dimensional object space, vehicles may follow two types of routes like static and dynamic. Trains generally follow static routes as they move through intermediate stations up to destination station. Their routes are fixed in the form of railway tracks. Public Transport usually uses fixed intra-city routes that are static. On the other side private vehicles follow random path; they are free to choose their path and hence their routes are dynamic.

The development and validation of the system in the present work has been carried out for the city of Jabalpur (India) and the public transport system here, is referred as Metro buses. In this paper, we present an extensible public vehicle position tracking system (PVPTS) that deploys data transmission capabilities of the Global System for Mobile Communication (GSM). Our design is extensible to bigger cities where many public vehicles are required to be simultaneously tracked and monitored. The local bus, train arrival information can be provided to the passengers using this system. A background of the relevant technologies used for tracking is given in Section 2. Existing technologies are discussed in Section 3; while section 4 contains methodolgy. Section 5 describes the implementation of PVPTS. Section 6 discusses on low cost solution. Section 7 shows system testing and results. Section 8 compares and contrasts this system with other PVPTS systems and concludes with a discussion on future directions for this research project.

\section{BACKGROUND}

The One of the possible reasons for people preferring private vehicles over public vehicles is that a passenger normally does not have exact information about the public vehicle arrival timing at their stops. By providing reliable public vehicle arrival information to the passengers at predefined stops, it is expected to improve the public vehicle occupancy. This is beneficial to both the passengers and public transport corporations. Tracking of a vehicle can be expressed as continuously monitoring of a vehicle. Tracking of buses can be useful for the automation of existing transportation systems [2]. By the use of tracking the information about bus-arrival timing can be easily provided to the passengers.

The different wireless technologies available today have resulted in the reliable and faster communication. GSM is an open, digital cellular technology used for transmitting mobile voice and data services [3]. GSM has become the world's fastest growing mobile communication standard. GSM networks operate in a number of different carrier frequency ranges (separated into GSM frequency ranges for $2 \mathrm{G}$ and UMTS frequency bands for $3 \mathrm{G}$ ), with most $2 \mathrm{G}$ GSM networks operating in the $900 \mathrm{MHz}$ or $1800 \mathrm{MHz}$ bands. The GSM network is structured into a number of discrete sections:

(i) The Base Station Subsystem (the base stations and their controllers).

(ii) The Network and Switching Subsystem (the part of the network most similar to a fixed network). This is sometimes also just called the core network.

(iii) The GPRS Core Network (the optional part which allows packet based Internet connections).

(iv) The Operations support system (OSS) for maintenance of the network.

Radio-Frequency Identification (RFID) is a wireless sensor technology which is based on the detection of electromagnetic signals [4]. There is emission of radio waves from the transmitter which can reach up to 100 feet or more, depending on its power output and the radio frequency 
used. RFID can be employed for different applications. RFID is used successfully in a variety of industries for different purposes. It is used for tagging and identifying the different items in a store. In the construction industry, RFID is used successfully for the tracking of pipe spools, structural steel members and as an on-site support system [5, 6].

\section{EXISTING TEChNOLOGIES}

There are many tracking applications available in the market for tracking of various public vehicles. One of the cost-effective technique for vehicle tracking is the 'vehicle card' technique [7], which uses STD phone booths and a specially designed vehicle-card. Vehicle owner is required to carry a card to the nearest tracking point, basically a designated STD booth on vehicle routes, at major vehicle stops and petrol bunks. The card is coded with details of the vehicle; these details and a simple numbered message are transferred to a local telephone number. The receiver uploads all incoming messages by email to a central server, where it is processed and placed on a website. In this way a vehicle can be tracked. But this scheme is not so useful for a large scale implementation of a fully automated real-time system. Another scheme for tracking has been proposed using odometer and inertial sensors [8]. But this technique has been proposed specifically for train transport. It does not gives a generalized solution for all the public transports. Similarly, there is one more technique employing distance-meter (odometer) and cellular infrastructure which is used for tracking vehicles in public domain [9].

One popular technique on which most of the moving objects tracking schemes rely is GPS (Global Positioning System) [10]. Most modern vehicle tracking systems use GPS modules which is costly in usage and implementation. GPS provides specially coded satellite signals that can be processed in a GPS receiver, enabling the receiver to compute position, velocity and time. Four GPS satellite signals are used to compute positions in three dimensions and the time offset in the receiver clock. GPS is the most accurate and popular positioning system. It provides a position accuracy of around 10 meters. A clear line of sight to at least four GPS satellites is necessary for high accuracy. GPS was developed by the U.S. Department of Defense. The system consists of a constellation of 24 satellites orbiting around 12,000 miles above the Earth's surface, shown in Figure 1. GPS was dedicated solely for military use and has recently been declassified for civilian use. The GPS receiver measures distances to four or more satellites simultaneously. GPS receivers take this information and use triangulation to calculate the user's exact location [11]. Factors that can degrade the GPS signal and thus affect accuracy include following:

(i) Ionosphere and troposphere delays - On passing through atmosphere the satellite signal slows down. Significantly larger delays occur for signals emitted from low elevation satellites.

(ii) Signal multi path - Satellite navigation receivers operate by line of sight with global positioning satellites. When GPS signal is reflected off big objects such as tall buildings or any large mountain before it reaches the receiver there is increase in the travel time of the signal, thereby causing errors.

(iii) Positioning errors - Generally, a satellite navigation receiver is accurate to within 10 $\mathrm{m}$. A number of positioning errors can occur, limiting accuracy to within $15-25 \mathrm{~m}$. There can be orbiting errors due to which a satellite's reported position does not match its actual trajectory.

One of the vehicle tracking technique used for vehicle tracking is Dead-Reckoning [12], in which the location of the vehicle is calculated by integrating the traveled distance in various directions in relation to a known initial location, often the previous location. The traveled distance is measured by wheel sensors; the driving direction is measured by an electronic compass. The 
wheel sensors are also used for determining the driving direction by frequently comparing the distance traveled by the left and the right wheel. Both obtained driving directions are compared to compensate for the accumulating error of the wheel sensors as well as for fluctuations in the earth magnetic field. As the measured distance and direction remain rough approximations, deadreckoning suffers from an accumulated error. The Travelpilot[13] is an automotive navigation system that is use the U.S and Europe that uses dead-reckoning and map-matching for navigation.

Evaluation of currently available technologies has found that each technology affords unique advantages for vehicle tracking applications while also facing distinctive challenges to overcome so as to satisfy the practical requirements in terms of reliable performance and cost effectiveness.

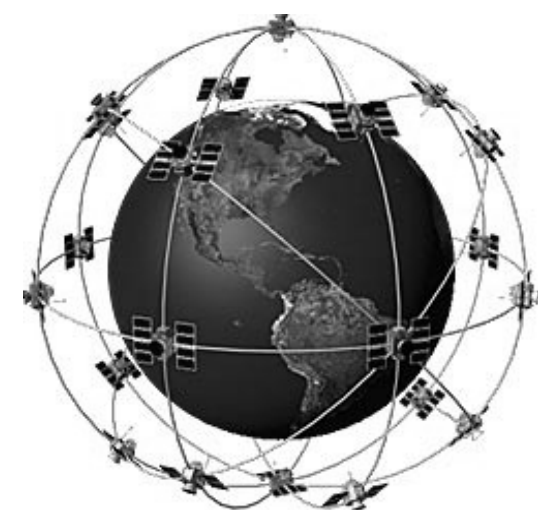

Figure 1. GPS consists of 24 satellites of which at least 5 can be seen from any point on the globe.

[10]

\section{MethodologY}

The tracking of any public vehicle involves the frequent and fast communication of vehicle's location information. For the purpose of vehicle tracking, information needs to be passed simultaneously at different locations so that passengers can get timely information. In all the techniques stated previously, either hardware or software overhead is often required at cell-sites. If vehicle routes under tracking are known in advance, a mapping system can easily trace a specific location on any route. A GIS can be used as a multimedia visualization tool for viewing and analyzing spatio-temporal relationships between different moving objects.

The proposed tracking system eliminates the need of additional hardware or software at cell-site. This proposed system depends upon GSM system and RFID for its operation. In our system, we have used the text-based data via the short message service enabled by the standard cellular phone communication network of GSM which provides a low-cost, effective solution in an urban area with an extensive coverage of mobile phone networks and has overcome the drawbacks of conventional walkie-talkie systems. The main limitations of data transmission on walkie-talkie or voice radios are:

(i) the possibility of losing data since data from multiple vehicles occupy the same radio channel.

(ii) the limited range the voice radio can cover. 
In proposed system 8 channel Radio transmitter and receiver with short range of few meters are used to get information about the presence of vehicle near the bus stop, which is further communicated through GSM module. In this way the system provides the information about the timing of bus arrival/departure in a semi-online manner. Table 1 compares the proposed scheme with other schemes such as Vehicle-card technique and Pure GPS/GPS-GPRS mixed.

Table 1. Comparison of vehicle tracking schemes

\begin{tabular}{|c|c|c|c|}
\hline \multirow[t]{2}{*}{ Parameter } & \multicolumn{3}{|c|}{ Technique Employed } \\
\hline & $\begin{array}{l}\text { Vehicle- } \\
\text { card } \\
\text { technique }\end{array}$ & $\begin{array}{l}\text { Pure } \\
\text { GPS/GPS- } \\
\text { GPRS } \\
\text { mixed }\end{array}$ & Proposed GSM and RFID technique \\
\hline $\begin{array}{l}\text { Accuracy of } \\
\text { location } \\
\text { detection }\end{array}$ & Lower & Accurate & Accurate \\
\hline $\begin{array}{l}\text { Cost of } \\
\text { installation }\end{array}$ & Very low & Highest & Much lesser than GPS based techniques \\
\hline $\begin{array}{l}\text { Suitability for } \\
\text { real-time } \\
\text { applications }\end{array}$ & $\begin{array}{l}\text { May be } \\
\text { adopted }\end{array}$ & Suitable & Suitable \\
\hline $\begin{array}{l}\text { Environmental } \\
\text { susceptibility } \\
\text { Scope of }\end{array}$ & $\begin{array}{l}\text { Not } \\
\text { applicable } \\
\text { High }\end{array}$ & $\begin{array}{l}\text { Moderate } \\
\text { Not at all }\end{array}$ & $\begin{array}{l}\text { Most Suited } \\
\text { Not at all }\end{array}$ \\
\hline $\begin{array}{l}\text { human } \\
\text { intervention }\end{array}$ & & & \\
\hline
\end{tabular}

The proposed system consists of two underlying technologies: GSM and RFID. Figure 2 shows the architecture of the proposed system. The PVPTS can be divided into three parts: Vehicle addressing and identification system, Information routing system and Display and data analysis system.

\subsection{Vehicle addressing and identification system}

Every vehicle is provided an identifier so that it can be recognized and tracked easily. Every vehicle has an 8 bit RF transmitter which is preset different for different vehicles. The RF transmitter always remains ON so that it can be identified by RF receivers located at every bus stop, whenever it is in proximity of few feet. The buses of same route may have same or different transmitting code. If buses of same route are given the same code we can track buses of 256 different bus routes simultaneously. In another case where particular public vehicle has to be tracked among the other vehicles of the same route, one or more bits out of 8 bits can be reserved for differentiating them.

\subsection{Information routing system:}

GSM is the most widely used among the digital wireless telephony technologies. The PVPTS uses Short Message Service (SMS) sent over GSM networks for routing information to different public vehicle stops. The signals from the GSM modem are sent to nearest Base Transceiver Station (BTS) that in turn are transferred to Mobile Switching Center (MSC) through Base Station Controller (BSC). The GSM modem equipment has a Subscriber Identity Module (SIM) security and authentication. The SIM is a detachable smart card containing the user's subscription 
information and phone book. The BTS and BSC together constitute the Base Station Subsystem (BSS) and perform all the functions related to the radio channel for data signaling and frequency hopping control and power level control. All of the elements in the system combine to produce many GSM services such as SMS which is used for information routing to different bus stops.

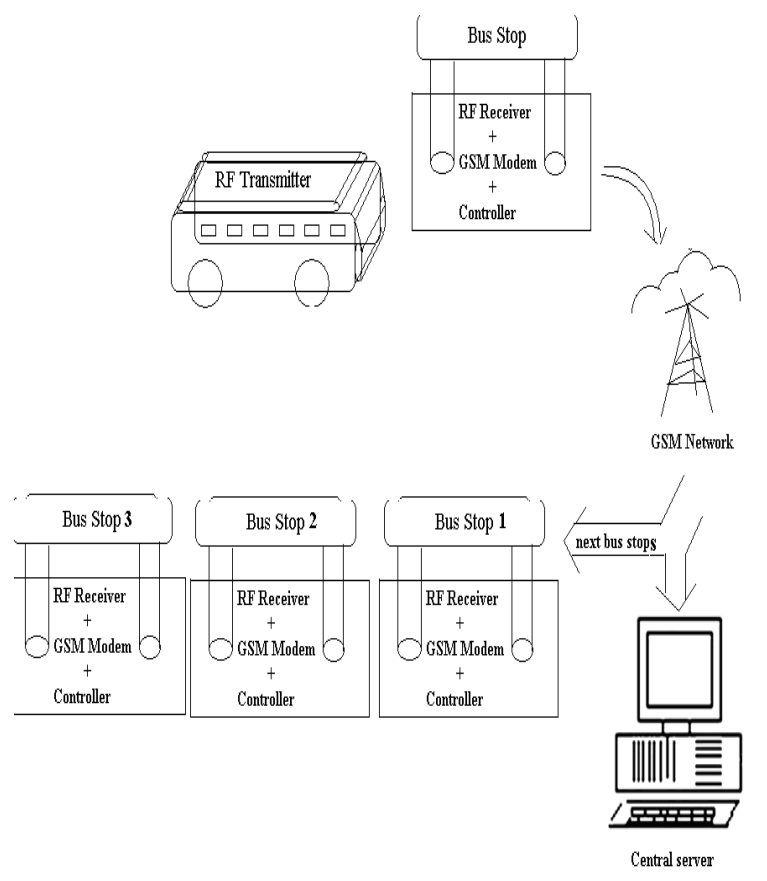

Figure 2. Architecture of the proposed system

\subsection{Vehicle addressing and identification system}

Display and data analysis system: LED displays are low cost, low power device capable of displaying text. LED display boards usually have clear visibility. LED display devices are placed at every bus stop, displaying the route number and time of arrival of a bus at bus stop. The vehicle location information received through GSM Modem placed at bus stop is sent to LED displays. The LED display periodically displays information of different buses along with time of arrival. Data stored at the central server can be easily analyzed through an application as It receives, processes, analyzes and stores the incoming SMS messages. The tracking of buses at central server thus can be easily carried out by having a GSM modem connected to server PC which updates the vehicle's location upon receiving the messages from different GSM modems placed at different bus stops.

\section{IMPLEMENTATION}

Several components are used in our tracking system. For identification, every vehicle has been provided with 8 bit RF transmitter. Different 8 bit words are transmitted by buses of different routes. The buses of same route may have same transmitting code if there are large numbers of public vehicles to be tracked in large cities. We have used the microcontroller unit which integrates RFID receiver and GSM unit placed at every bus stop. Every bus stop has 8 bit RF receiver along with GSM modem installed to send and receive data. Whenever any bus comes within the range of about 100 feet of the bus stop, the receiver receives the 8 bit word sent by the 
transmitter in a bus, which is kept continuously on. Thus information routing is done using the microcontroller unit which integrates RFID receiver and GSM unit placed at every bus.

The microcontroller units used at different bus stops are programmed such that they contain GSM number of the modems placed at further bus stops. The communication between microcontroller and modem is done using USART (Universal Synchronous Asynchronous Receiver and Transmitter). The interfacing of RF receiver data with GSM modem is done using microcontroller unit. The eight bits signal received at receiver are then sent to microcontroller which decodes it and depending on the bits received sends the AT commands to the GSM modem required for forwarding vehicle location information to central server and next stops. The algorithm used for various microcontroller operations is as follows,

\section{Setting the text command for configuring modem:}

1. Send " $\mathrm{AT}+\mathrm{CMGF}=1$ " $<\mathrm{CR}>$

2. Receive the characters from the modem and store in an array and check for "K".

3. If " $\mathrm{K}$ " is present, then text message is $\mathrm{OK}$.

4. Else, reinitialize the modem

5. Delay 1 second.

\section{Enabling for SMS:}

1. Send "AT+CNMI $=2,1,0,0,0$ " $<\mathrm{CR}>$

2. Receive the characters from the modem and store in an array and check for " $K$ ". If " $K$ " is present then modem is initialized completely.

3. Else goto reinitialize the modem.

\section{Sending status to other stops:}

1. Send "AT+CMGS=n" $<\mathrm{CR}>$ where $\mathrm{n}$ is next bus stops phone number.

2. Send the bus identity message along with estimated time for arrival to next 'n' stops.

3. Send 0x1A.

\section{Receiving the SMS Number from the modem:}

1. Keep on receiving bytes until ' + 'comes.

2. When ' + ' comes then start storing the bytes in array up to $12^{\text {th }}$ byte.

3. The $12^{\text {th }}$ byte is the SMS No.

4. Whenever a SMS is received we receive AT+CMTI: "SM", 1 .

\section{Reading the SMS Received:}

1. Send " $\mathrm{AT}+\mathrm{CMGR}=$ " $<\mathrm{CR}>$ to check for any incoming message 
2. Give delay of 1 millisecond.

3. Send the SMS Number.

4. Send 0x0D.

5. Send 0x0A.

6. Receive bytes until ' 1 ' comes.

7. After getting ' 1 ' start storing the received bytes in an array until " comes.

8. This is the sender's phone number.

9. Continue receiving until $*$ comes.

10. Start storing the character after $* \&$ store in an array until \# comes.

11. If the array size is below 25 , then it is a valid message

\section{Deleting the SMS :}

1. Set "AT+CMGD="

2. Delay $1 \mathrm{msec}$.

3. Send SMS number.

4. Send 0x0D.

5. Send 0x0A.

6. Keep on receiving characters from the modem till ' $\mathrm{K}$ ' comes.

7. When ' $\mathrm{K}$ ' is received, SMS is deleted.

'AT' commands sent to GSM modem forwards the SMS containing the 8 bit code of the bus and bus stop id to the next 4 or more bus stops and to central server. Thus, the information about location of bus is passed to next bus stops. We have designed, implemented and tested the system in our lab. The system prototype set up and some of its components are shown in Figure 3. To test the functionality of the system we placed the RF transmitter in a mobile vehicle. While the vehicle was moving vehicle's current location was reported to the further stops as well as to the central server. The SMS received by the modems located at different bus-stops is sent to the microcontroller unit. The microcontroller unit analyzes the received message through GSM modem and decodes the information being sent in the form of message. Finally, information about bus arrival is sent to the LED display board connected to microcontroller unit. The LED display board displays the 
International Journal of UbiComp (IJU), Vol.3, No.1, January 2012

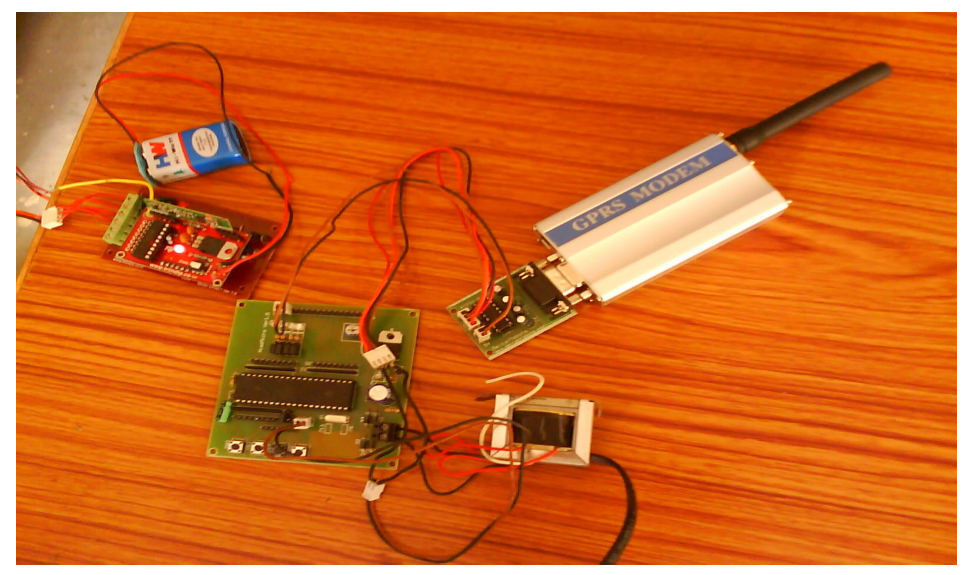

Figure 3. RF Receiver and GSM/GPRS modem used for lab testing

message about bus with estimated time of arrival of bus at that bus stop. The LED boards display the time of arrival of different buses as different messages are sent to it through the microcontroller unit. Some useful information can also be displayed on the display boards as message can be also be forwarded from the central server having database of phone numbers allotted to different SIM placed in the GSM modems. Thus, important information like route change due to some reasons, can be circulated to the public in different situations.

\section{Discussion ON Low COST SOLUTION}

Efforts have been taken to reduce the total cost of the system including devices and services. Starting from small transport systems to larger transport systems, the devices and services cost can be made affordable. The different components used are 8 channel Radio frequency transmitter and receiver, GSM modem, interfacing controller unit and display unit which make the overall system cost effective. By using SMS for communication, the service cost has been reduced drastically. Most operators are providing SMS services at very cheap rates. Thus components and service cost of the system is much lesser than other tracking systems available in market.

\section{SYSTEM TESTING AND RESULTS}

System design is verified by testing after integration of all components of the system. The whole setup consisting of various components like RFID transmitter and receiver, GSM modem along with controller interface is tested in lab. Debugging serial port of microcontroller was connected to a PC's COM port to see the results on HyperTerminal during its operation as shown in the Figure 4. 


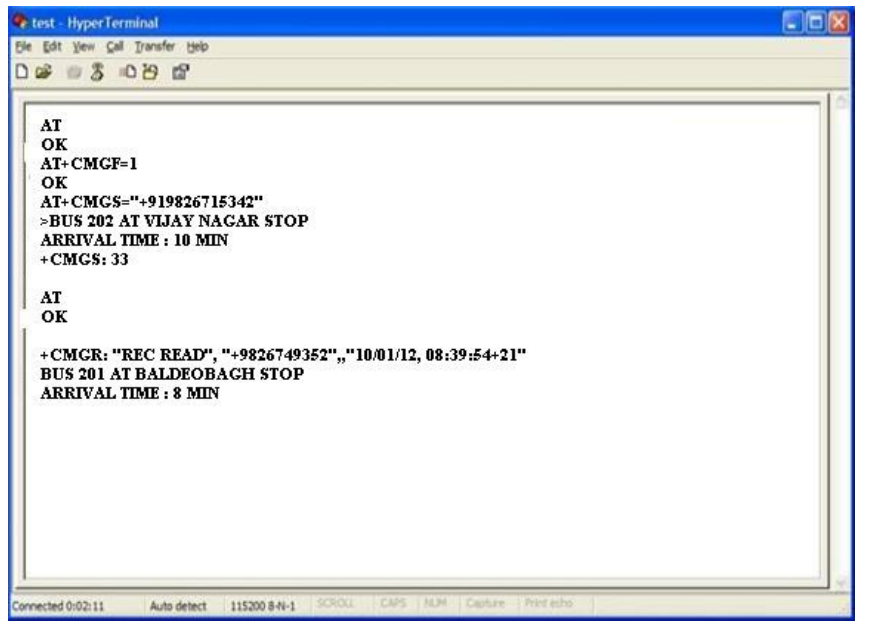

Figure 4. Hyperterminal image showing simulation results

The LED/LCD board displays the location of bus with estimated time of arrival of bus at that bus stop, as shown in Figure 5. The tracking of buses at some server located at a particular place is easily carried out by having a modem connected to server. The location information of different buses is updated, as GSM modems located at each bus stop forwards an SMS containing vehicle location information to this central server. Thus the central server keeps tracks of different buses. Figure 6 shows the application interface build using Microsoft Visual Basic at the server. It receives the different messages, thus keeps track of public vehicle's current location. The application interface receives and analyzes the data received by it and thus tracking of all the buses can be done at the central server providing more information which can be used for analysis of traffic in different routes and other information.

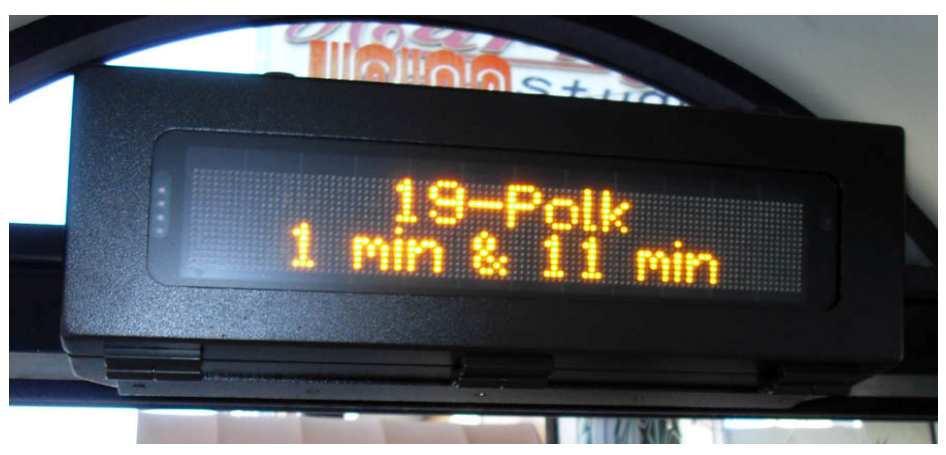

Figure 5. LED display unit 


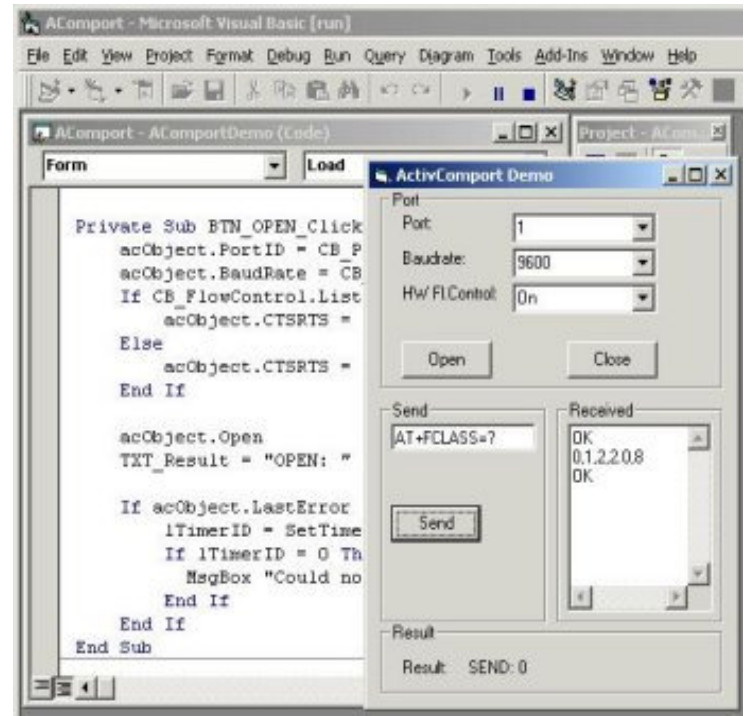

Figure 6. Application interface at the server

\section{CONCLUSION}

The key feature of this system is its relatively simple mode of communication. The tracking of different local buses or local train can be done easily. The project involves the use of GSM and RFID technology which is used to send and receive information for locating any vehicle. Our proposed system is vulnerable to network congestions as the SMS sent to other locations may get delayed. Thus, the efficiency of the proposed system depends on the congestion free working of GSM networks. The use of GSM technology as compared to other existing techniques used for tracking makes it more cost efficient and innovative. Our system design has an open architecture that can be easily expanded to other applications. These features make the device completely suitable for public vehicle tracking applications because:

(i) low cost system opens up a new segment of the market.

use of GSM technology allows tracking in high density urban areas

This system can be easily extended for central tracking system to keep track of all the public vehicles. The code size of microcontrollers can be reduced as the vehicle's location information routing can be done via central server application. The different queries and efficient route management can be easily done through central server system. Trends in wireless technology have resulted in most of the passengers owning mobile phones. We believe that by providing busroute and arrival information to passengers' cell phones in a timely manner, it is possible to improve the bus occupancy. Thus, this system can be integrated with internet which maintains the vehicle's current location database so that people owing mobiles can enter vehicle route number and bus-stop ID and get the time arrival of respective bus on their mobile. Due to easily available gadgets like mobile phones, laptops, Personal Digital Assistants (PDAs), passengers would not have to rely on old manual enquiry terminals. Thus, the proposed system is beneficial for both passengers and the bus operators. 


\section{REFERENCES}

1. Figueiredo, L., Jesus, I., Machado, J.A.T., Ferreira, J.R., Martins de Carvalho, J.L., "Towards the Development of Intelligent Transportation Systems". IEEE Intelligent Transportation Systems Proceedings, Oakland, CA (2001)

2. Lee, K., Ryu, H.Y., "Automatic circuity and accessibility extraction by road graph network and its application with high resolution satellite imagery", Proceedings of the IEEE International Geosciences and Remote Sensing Symposium, IGARSS'04, vol. 5, 2024, September 2004, pp. 3144-3146 (2004)

3. GSM - Wikipedia, the free encyclopedia, http:// http://en.wikipedia.org/wiki/GSM

4. Landt, J., "The history of RFID," IEEE Potentials, Vol. 24, No. 4, 8-11, (2005)

5. Domdouzis, K., Kumar, B., Anumba, C., "Radio-Frequency Identification (RFID) applications: A brief introduction", Advanced Engineering Informatics, v.21 n.4, p.350$355,(2007)$

6. Kim, M., Chong, N.Y., "RFID-based mobile robot guidance to a stationary target", Mechatronics, 17(4-5), pp.217-229, Elsevier, (2007)

7. Rus, C., Bilcu, R.C., Egiazarian, K., Rusu, C., "Scanned maps processing using wavelet domain hidden Markov models", First International Symposium on Control, Communications and Signal Processing, 2004, pp. 451-454. IEEE, (2004)

8. Ernest, P., Mazl, R., Preucil, L., "Train locator using inertial sensors and odometer", IEEE Intelligent Vehicles Symposium 2004, 14-17, June 2004, pp. 860-865 (2004)

9. Kane, L., Verma, B., Jain, S., "Vehicle tracking in public transport domain and associated spatio-temporal query processing", Elsevier: Computer Communications, v.31 n.12, p.2862-2869 (2008)

10. Garmin I What is GPS?, http://www.garmin.com/aboutGPS/index.html

11. Global Positioning System Overview, http://www.colorado.edu/geography/gcraft/notes/gps/gps.html

12. IVHS: Positioning and Navigation, http://www.wirelesscommunication.nl/reference/chaptr01/roadtrin/positi.htm

13. Buxton, J. L.,Honey,S. K., Suchowerskyj,W. E., Tempelhof, A., The Travelpilot: a second-generation automotive navigation system by, Vehicular Technology, IEEE Transactions on In Vehicular Technology, Vol. 40, No. 1. (1991), pp. 41-44 